\title{
Diffractive vector meson production at HERA using holographic AdS/QCD wavefunctions
}

\author{
Jeff FORSHAW \\ University of Manchester \\ E-mail: jeff.forshaw@hep.manchester.ac.uk \\ Ruben SANDAPEN ${ }^{*}$ \\ Université de Moncton \& Mount Allison University \\ E-mail: ruben. sandapen@umoncton. ca
}

\begin{abstract}
We demonstrate another success of the AdS/QCD correspondence by showing $[1,2]$ that an AdS/QCD holographic light-front wavefunction for the $\rho$ meson generates predictions for the cross-sections of diffractive $\rho$ production that are in agreement with data collected at the HERA electron-proton collider [3, 4].
\end{abstract}

XXI International Workshop on Deep-Inelastic Scattering and Related Subjects 22-26 April, 2013

Marseilles, France

\footnotetext{
* Speaker.

${ }^{\dagger} \mathrm{RS}$ thanks the organisers for their invitation as well as Université de Moncton \& Mount Allison University for funding.
} 


\section{Introduction}

The AdS/QCD correspondence [5, 6, 7, 8] refers to the connection between QCD in physical spacetime and string theory in a higher dimensional anti-de Sitter (AdS) space. The precise nature of this connection has not yet been elucidated but there is growing evidence, to which we add here, that there exists such a connection. One particular realization of this connection is light-front holography [9] proposed by Brodsky and de Téramond. In light-front holography, the confining QCD potential at equal light-front time between a quark and antiquark in a meson is determined by the profile of the dilaton field which breaks conformal invariance of the higher dimensional AdS space in which strings propagate.

In a semi-classical approximation to light-front QCD, Brodsky and de Téramond derived a Schroedinger-like equation for mesons:

$$
\left(-\frac{\mathrm{d}^{2}}{\mathrm{~d} \zeta^{2}}-\frac{1-4 L^{2}}{4 \zeta^{2}}+U(\zeta)\right) \Phi(\zeta)=M^{2} \Phi(\zeta)
$$

where $\zeta=\sqrt{x(1-x)} r$ is the transverse separation between the quark and antiquark at equal lightfront time ${ }^{1}, L$ is the orbital quantum number, $M$ is the mass of the meson and $\Phi(\zeta)$ is the transverse mode of the light-front wavefunction which is itself given by

$$
\phi(x, \zeta, \varphi)=\frac{\Phi(\zeta)}{\sqrt{2 \pi \zeta}} f(x) \mathrm{e}^{i L \varphi}
$$

It remains a challenge to derive the confining potential $U(\zeta)$ from first-principles QCD but after identifying $\zeta$ with the co-ordinate in the fifth dimension and angular momentum with the fifth dimensional mass ${ }^{2}$, equation (1.1) describes the propagation of spin- $J$ string modes, in which case $U(\zeta)$ is determined by the choice for the dilaton field. Remarkably, it can be shown [10] that the dilaton profile is constrained to be quadratic so that the resulting confining potential is given by

$$
U(\zeta)=\kappa^{4} \zeta^{2}+2 \kappa^{2}(J-1)
$$

Solving equation (1.1) with this confining potential yields the eigenfunctions

$$
\Phi_{n L}(\zeta)=\kappa^{1+L} \sqrt{\frac{2 n}{(n+L)}} \zeta^{1 / 2+L} \exp \left(-\kappa^{2} \zeta^{2} / 2\right) L_{n}^{L}\left(\kappa^{2} \zeta^{2}\right)
$$

with the corresponding eigenvalues

$$
M_{n L, S}^{2}=4 \kappa^{2}\left(n+L+\frac{S}{2}\right)
$$

\footnotetext{
${ }^{1} x$ is the fraction of light-front momentum carried by the quark and $r$ is the transverse separation between the quark and the antiquark at equal ordinary time

${ }^{2}\left(m_{5} R\right)^{2}=-(2-J)^{2}+L^{2}$ where $R$ is the radius of curvature in AdS space.
} 


\section{The $\rho$ meson wavefunction}

For the $\rho$ meson, $n=1, L=0$ and $J=1$ so that $\kappa=M_{\rho} / \sqrt{2}=0.54 \mathrm{GeV}$. In equation (1.2), $f(x)$ is fixed by comparing the expressions for the pion EM form factor in light-front QCD and in AdS space. This yields $f(x)=\sqrt{x(1-x)}$ so that the resulting AdS/QCD for the $\rho$ is then given by

$$
\phi(x, \zeta) \propto \sqrt{x(1-x)} \exp \left(-\frac{\kappa^{2} \zeta^{2}}{2}\right) \exp \left(-\frac{m_{f}^{2}}{2 \kappa^{2} x(1-x)}\right)
$$

where the dependence on the quark mass has been introduced according to the prescription by Brodsky and de Téramond [11]. Here we use a light quark mass $m_{f}=0,14 \mathrm{GeV} \mathrm{[1].}$

An earlier procedure to obtain the meson wavefunction is by boosting a non relativistic gaussian Schroedinger wavefunction $[12,13]$ which results in the so-called Boosted Gaussian (BG):

$$
\phi^{\mathrm{BG}}(x, \zeta) \propto x(1-x) \exp \left(\frac{m_{f}^{2} R^{2}}{2}\right) \exp \left(-\frac{m_{f}^{2} R^{2}}{8 x(1-x)}\right) \exp \left(-\frac{2 \zeta^{2}}{R^{2}}\right) .
$$

If $R^{2}=4 / \kappa^{2}$ then the two wavefunctions differ only by a factor of $\sqrt{x(1-x)}$, which is not surprising given that in both cases confinement is modelled by a harmonic oscillator [1]. In what follows we shall consider a parameterization that accommodates both the AdS/QCD and the BG wavefunctions:

$$
\phi(x, \zeta) \propto[x(1-x)]^{\beta} \exp \left(-\frac{\kappa^{2} \zeta^{2}}{2}\right) \exp \left(-\frac{m_{f}^{2}}{2 \kappa^{2} x(1-x)}\right) .
$$

The AdS/QCD wavefunction is obtained by fixing $\beta=0.5$ and $\kappa=0.55 \mathrm{GeV}$ where as the $\mathrm{BG}$ wavefunction is obtained by fixing $\beta=1$ and treating $\kappa$ as a free parameter.

\section{Results and conclusions}

To compute the rate for diffractive $\rho$ production, we use the dipole model of high-energy scattering $[14,15,16,17]$ in which the scattering amplitude for diffractive $\rho$ meson production is a convolution of the photon and vector meson $q \bar{q}$ light-front wavefunctions with the total crosssection to scatter a $q \bar{q}$ dipole off a proton. QED is used to determine the photon wavefunction and the dipole cross-section can be extracted from the precise data on the deep-inelastic structure function $F_{2}[18,19]$. This formalism can then be used to predict rates for vector meson production and diffractive DIS $[13,20]$ or to to extract information on the $\rho$ meson wavefunction using the HERA data on diffractive $\rho$ production $[21,22]$. Here we use it to test whether the HERA data prefer the AdS/QCD wavefunction given by equation (2.1). To do so, we compute the $\chi^{2}$ per data point in the $(\beta, \kappa)$ parameter space using the parametrization (2.3) for the $\rho$ wavefunction. ${ }^{3}$

Figure 1 confirms that the AdS/QCD prediction lies impressively close to the minimum in $\chi^{2}$. The best fit has a $\chi^{2}$ per data point equal to $114 / 76$ and is achieved with $\kappa=0.56 \mathrm{GeV}$ and $\beta=0.47$ which should be compared with the AdS/QCD prediction: $\kappa=0.54$ and $\beta=0.5$ shown

\footnotetext{
${ }^{3}$ We include the electroproduction data and decay width datum in the fit.
} 


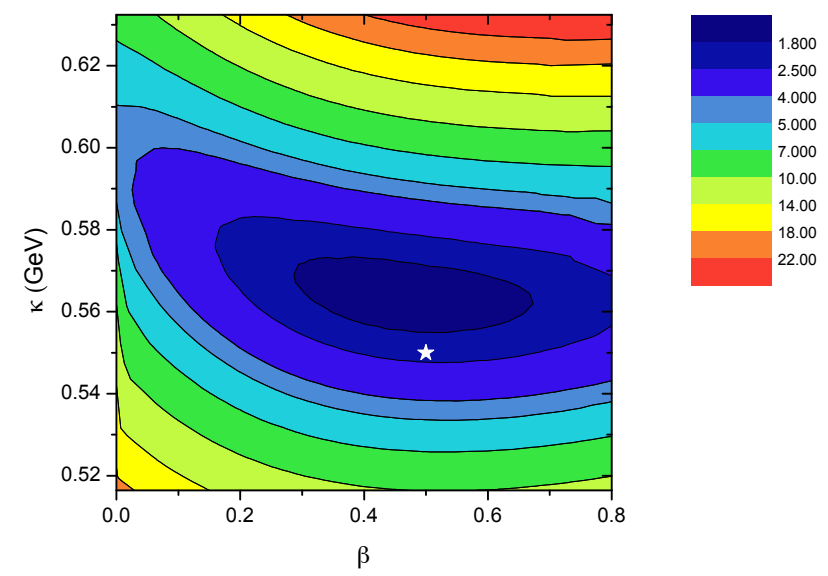

Figure 1: The $\chi^{2}$ distribution in the $(\beta, \kappa)$ parameter space. The AdS/QCD prediction is the white star.

as the white star on figure 1 . Note that the $\mathrm{BG}$ prediction i.e. $\beta=1, \forall \kappa$, is clearly further away from the minimum in $\chi^{2}$.

Finally, we note that these results are produced using a particular Color Glass Condensate dipole model [18] but that similar results are obtained by using other forward dipole models [19] that fit the $F_{2}$ structure function data. It remains to be seen how the $\chi^{2}$ distribution changes if a more sophisticated dipole model, such as the recent impact parameter saturation model [23] which fits the combined HERA $F_{2}$ data, is used.

\section{References}

[1] J. R. Forshaw and R. Sandapen, An AdS/QCD holographic wavefunction for the rho meson and diffractive rho meson electroproduction, Phys.Rev.Lett. 109 (2012) 081601, [arXiv: 1203.6088 ].

[2] J. R. Forshaw and R. Sandapen, Diffractive rho production with an AdS/QCD holographic wavefunction for the rho meson, AIP Conf.Proc. 1523 (2012) 87-90, [arXiv:1211.4729].

[3] ZEUS Collaboration, S. Chekanov et. al., Exclusive $\rho^{0}$ production in deep inelastic scattering at HERA, PMC Phys. A1 (2007) 6, [arXiv:0 708.1478$].$

[4] The H1 Collaboration, Diffractive Electroproduction of $\rho$ and $\phi$ Mesons at HERA, JHEP 05 (2010) 032, [arXiv:0910.5831].

[5] J. Erdmenger, N. Evans, I. Kirsch, and E. Threlfall, Mesons in Gauge/Gravity Duals - A Review, Eur.Phys.J. A35 (2008) 81-133, [arXiv: 0711.4467$].$

[6] J. Casalderrey-Solana, H. Liu, D. Mateos, K. Rajagopal, and U. A. Wiedemann, Gauge/String Duality, Hot QCD and Heavy Ion Collisions, arXiv: 1101.0618.

[7] M. S. Costa and M. Djuric, Deeply Virtual Compton Scattering from Gauge/Gravity Duality, arXiv:1201.1307.

[8] G. F. de Teramond and S. J. Brodsky, Hadronic Form Factor Models and Spectroscopy Within the Gauge/Gravity Correspondence, arXiv:1203.4025. 
[9] G. F. de Teramond and S. J. Brodsky, Light-Front Holography: A First Approximation to QCD, Phys.Rev.Lett. 102 (2009) 081601, [arXiv: 0809.4899 ].

[10] S. J. Brodsky, G. F. de TÃl'ramond, and H. G. Dosch, Conformal Symmetry, Confinement, and Light-Front Holographic QCD, arXiv:1302.5399.

[11] S. J. Brodsky and G. F. de Teramond, Light-Front Holography and Novel Effects in QCD, AIP Conf.Proc. 1116 (2009) 311-326, [arXiv:0812.3192].

[12] J. Nemchik, N. N. Nikolaev, E. Predazzi, and B. G. Zakharov, Color dipole phenomenology of diffractive electroproduction of light vector mesons at HERA, Z. Phys. $\mathbf{C 7 5}$ (1997) 71-87, [hep-ph/9605231].

[13] J. R. Forshaw, R. Sandapen, and G. Shaw, Colour dipoles and $\rho$, $\phi$ electroproduction, Phys. Rev. D69 (2004) 094013, [hep-ph/0312172].

[14] N. N. Nikolaev and B. G. Zakharov, Colour transparency and scaling properties of nuclear shadowing in deep inelastic scattering, Z. Phys. C49 (1991) 607-618.

[15] N. N. Nikolaev and B. G. Zakharov, Pomeron structure function and diffraction dissociation of virtual photons in perturbative QCD, Z. Phys. C53 (1992) 331-346.

[16] A. H. Mueller, Soft gluons in the infinite momentum wave function and the BFKL pomeron, Nucl. Phys. B415 (1994) 373-385.

[17] A. H. Mueller and B. Patel, Single and double BFKL pomeron exchange and a dipole picture of high-energy hard processes, Nucl. Phys. B425 (1994) 471-488, [hep-ph/ 9403256 ].

[18] G. Soyez, Saturation QCD predictions with heavy quarks at HERA, Phys. Lett. $\mathbf{6 6 5 5}$ (2007) 32-38, [arXiv:0705.3672].

[19] J. R. Forshaw and G. Shaw, Gluon saturation in the colour dipole model?, JHEP 12 (2004) 052, [hep-ph/0 411337].

[20] J. R. Forshaw, R. Sandapen, and G. Shaw, Further success of the colour dipole model, JHEP 11 (2006) 025, [hep-ph/0608161].

[21] J. R. Forshaw and R. Sandapen, Extracting the rho meson wavefunction from HERA data, JHEP 11 (2010) 037, [arXiv:1007.1990].

[22] J. R. Forshaw and R. Sandapen, Extracting the Distribution Amplitudes of the rho meson from the Color Glass Condensate, JHEP 1110 (2011) 093, [arXiv: 1104 . 4753].

[23] A. H. Rezaeian, M. Siddikov, M. Van de Klundert, and R. Venugopalan, Analysis of combined HERA data in the Impact-Parameter dependent Saturation model, Phys.Rev. D87 (2013) 034002, [arXiv:1212.2974]. 